\title{
Analysis of Tea Geometrid (Ectropis grisescens) Pheromone Gland Extracts Using
}

\section{GC-EAD and GC $\times$ GC/TOFMS}

Tao $\mathrm{Ma}^{1}$, Qiang Xiao ${ }^{2}$, Yu-Geng Yu ${ }^{2}$, Cai Wang $^{1}$, Cheng-Qi Zhu ${ }^{1}$, Zhao-Hui Sun ${ }^{1}$, Xiao-Yang Chen ${ }^{1}$, Xiu-Jun Wen ${ }^{1 *}$

1. Guangdong Key Laboratory for Innovative Development and Utilization of Forest Plant Germplasm, College of Forestry and Landscape Architecture, South China Agricultural University, Guangzhou, 510642, China

2. Key Laboratory of Tea Plants Biology and Resources Utilization of Agriculture Ministry, Tea Research Institute, Chinese Academy of Agricultural Sciences, Hangzhou, 310008, China

\section{Corresponding Author}

Telephone/Fax: +86-020-85280256, E-mail: wenxiujun@scau.edu.cn 


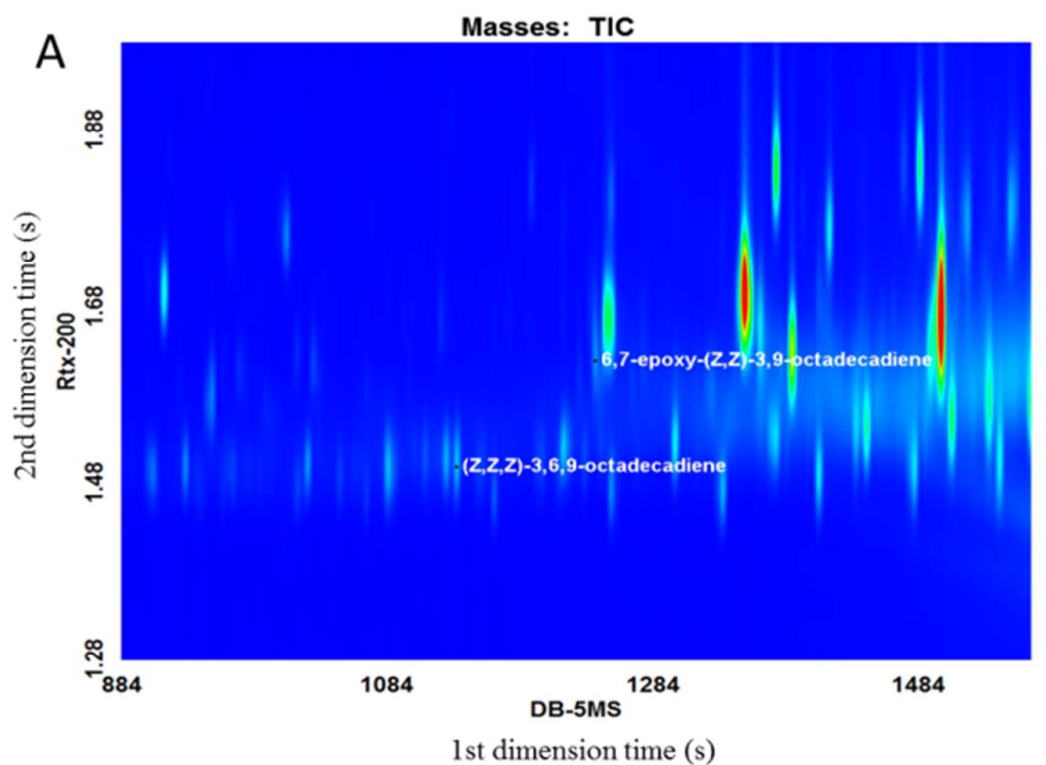

B

Masses: TIC

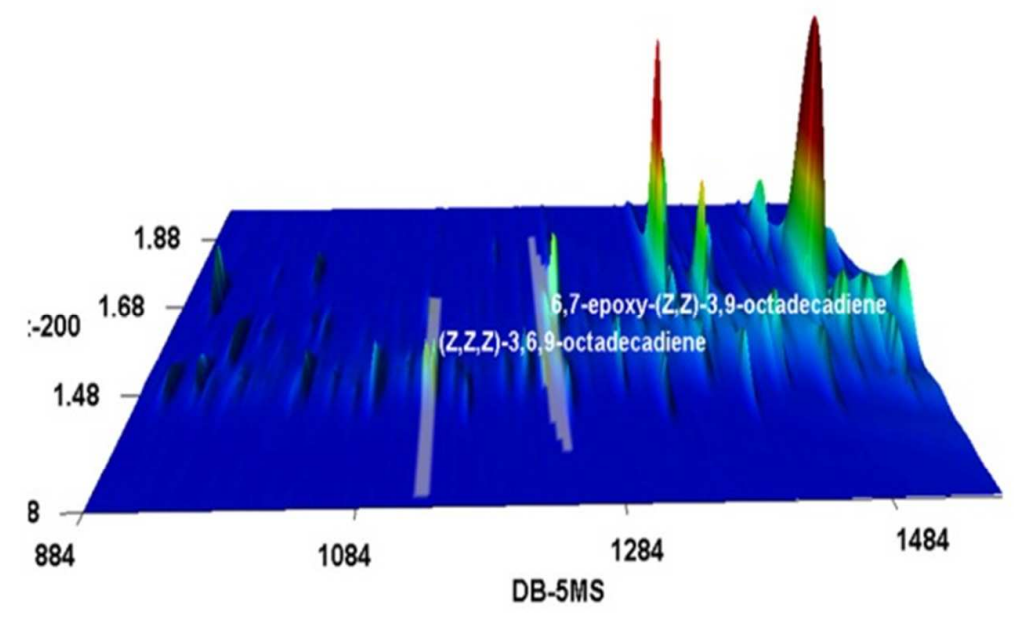




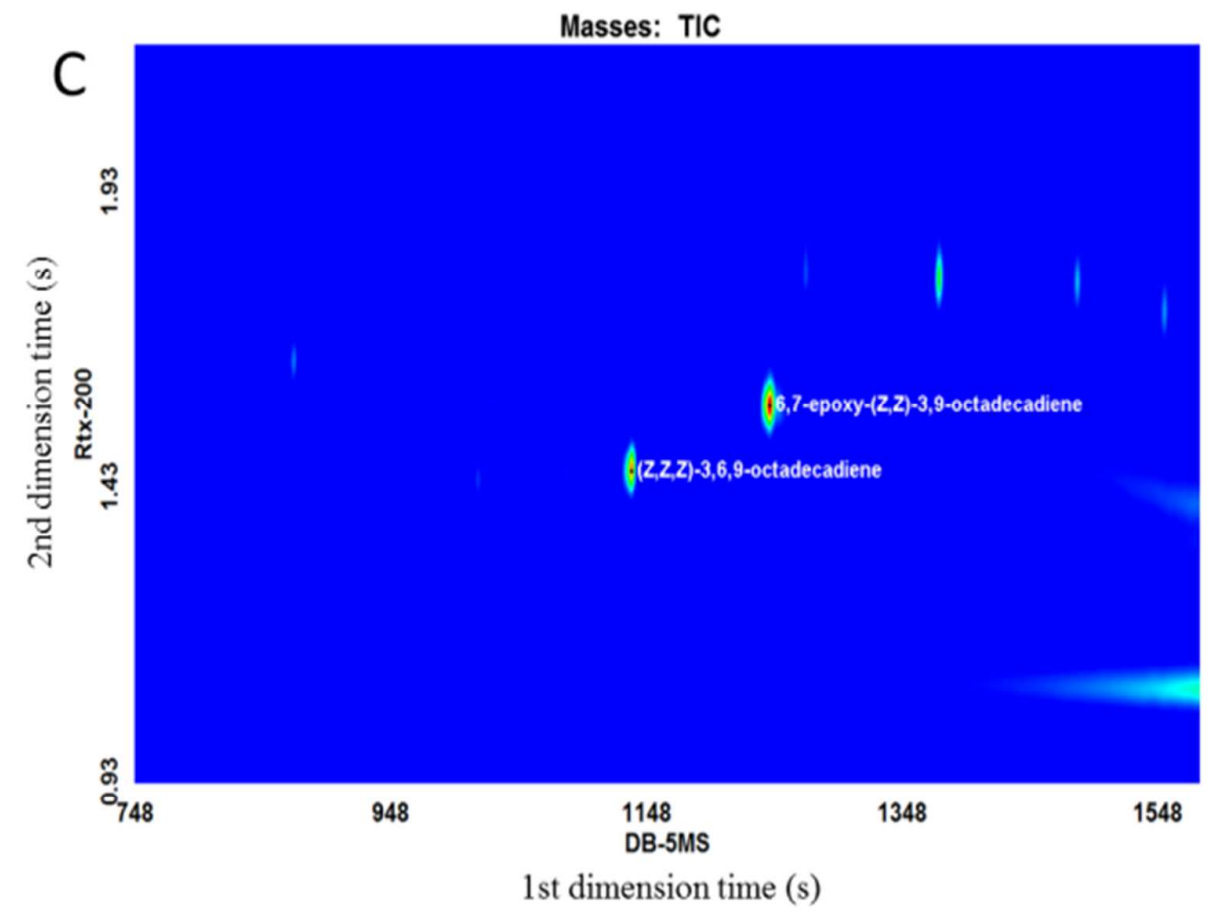

D Masses: TIC

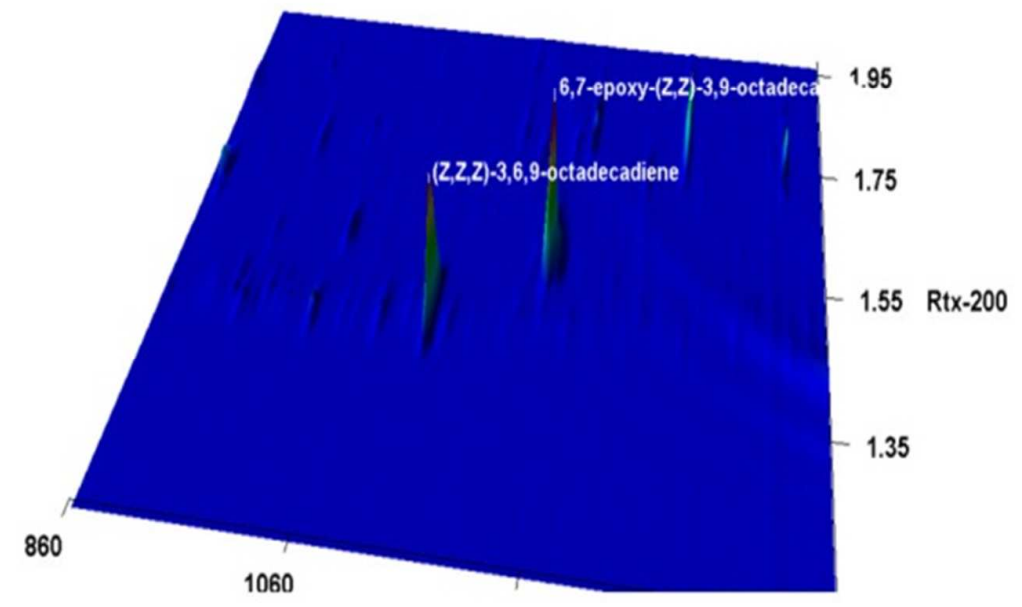

Figure S1. GCXGC/TOFMS chromatogram from female pheromone gland extracts and synthetic compounds (2D and 3D surface plots). A and B: Analysis of pheromone gland extracts; C and D: Analysis of synthetic compounds. 